\title{
Etat des connaissances et conservation de flore endemique dans la region d'Oran (Algerie occidentale)
}

Sihem Mansouri', Mohamed Djamel Miara² \& Seghir Hadjadj-Aoul ${ }^{1}$

'Laboratoire d'Ecologie. Département de Biologie. Université d'Oran 1, Ahmed Ben Bella. 31000, DZ.

2Département de Biologie. Faculté SNV. Université Ibn Khaldoun. Tiaret. Algerie. 14000, DZ.

\section{Correspondencia}

M. D. Miara

e-mail: miara14130@yahoo.fr

Recibido: 3 septiembre 2018

Aceptado: 18 septiembre 2018

Publicado on-line: noviembre 2018

\begin{abstract}
Résumé
Cette étude est une première exploration régionale visant la recherche des plantes endémiques de la région d’Oran en vue d’une éventuelle évaluation de leur état de conservation. Les recherches se sont basées essentiellement sur les 124 relevés phytoecologiques réalisés à travers les secteurs 01 et O2 ainsi que certains sorties ciblant les stations de présence des espèces endémiques signalées pour la région d Oran dans la littérature. Ces recherches de terrain ont conduit à l,identification de 17 taxons endémiques dont certains sont très rares comme Brassic aspinescens et Helianthemum maritimum. D'autres espèces ayant été ciblées par les recherches n'ont pas été retrouvées ce qui renforce l'hypothèse de disparition de certaines d'entre elles. En plus de cette première liste des endémiques réellement observés dans la région depuis l'indépendance du pays, les données sur leurs aspects chorologiques, écologiques et biogéographiques sont exposées et discutés.
\end{abstract}

Mots clés: Endémique, flore, chorologie, conservation, inventaire, Oran, rare.

\begin{abstract}
State of knowledge and conservation of endemic flora in the region of Oran (Western Algeria)

This study is a first regional exploration for plants research of the Oran region for a possible evaluation for their conditions of conservation. The research were basically based on 124 phytoecological records carried out across the sectors $\mathrm{O} 1$ and $\mathrm{O} 2$ as well as some data targeting endemic species presence stations reported for the region of Oran in the literature. This field searches led to the identification of 17 endemic taxa some of which are very rare like Brassica spinescens and Helianthemum maritimum. Other targeted species were not found, which reinforces the hypothesis of the disappearance of some of them. In addition to this first list of endemics actually observed in the region since the independence of the country, the data on their chorological, ecological and phytobiogeographical aspects are exposed and discussed.
\end{abstract}

Key words: Endemics, flora, chorology, conservation, inventory, Oran, rare.

\section{Introduction}

La région méditerranéenne actuelle figure en seconde position parmi les 34 hotspots mondiaux définis par Myers (1988). Cette région écobiogéographique (écorégion) présente une biodiversité exceptionnelle d'environ 25000 plantes vasculaires (Quézel ,1986), voire 28000 à 30000 espèces et sous-espèces (Greuter, 1995 ; Heywood, 1998), et représente un centre majeur d'endémisme avec un très grand nombre de genres endémiques (150) et d'espèces endémiques (13000 soit environs 50\%) (Quézel, 1956 ; Mittermeier et al., 1998 ; Rivas-Martinez \& Loidi Arregui ,1999).

La flore algérienne compte 3139 espèces répartis dans près de 150 familles parmi lesquelles 653 espèces sont endémiques, soit un taux de 12,6 $\%$ d'endémisme. En ne considérant que le secteur oranais, celui-ci conserve environ 1780 espèces végétales du total de la flore algérienne soit environ $57 \%$, ainsi que $95 \%$ de la flore méditerranéenne 
maghrébine comptant 1865 espèces (Quézel, 2000). D'après Véla \& Benhouhou (2007), la région d'Oran notamment les zones côtières figure parmi les secteurs les plus remarquables pour l'endémisme en Algérie.

Les premiers travaux visant à identifier les zones importantes pour les plantes en Algérie ont permis de classer les collines d'Oran comme une zone importante pour les plantes car elle renferme plusieurs taxons endémiques menacés (Yahi et al., 2012).

En effet, cette région incluant les deux secteurs biogéographiques (O1 et O2) sensu Quézel \& Santa (1962-63) abrite 177 taxons endémiques (Algérie, Algérie-Maroc et Algérie-Tunisie) (Véla \& Benhouhou, 2007). Parmi ces taxons, 3 espèces figurant dans la liste rouge de I'UICN (Walter \& Gillett, 1998).

Les données issues de cette première phase de définition des principales ZIP en Algérie (Yahi et al., 2012) étaient plutôt basées sur la bibliographie ancienne notamment la flore d'Algérie datant de 1962 qui présente désormais beaucoup de problèmes de nomenclature et de chorologie et semble avoir besoin d'une sérieuse vérification et mise à jours. Mis à part les changements taxonomiques et chorologiques qu'ont subits les espèces de cette flore (Dobignard \& Chatelain, 2010-13), la situation actuelle de la plupart des taxons endémiques est mal connue. En effet, la majorité des espèces endémiques algériennes n'ont pas été revues depuis leurs description durant l'époque coloniale ce qui laisse à se poser des questions par rapport à leurs statut d existence à la lumières des fortes modifications que leurs écosystèmes et habitats naturels ont subits (Miara et al., 2017).

C'est ainsi que la prochaine étape du travail consiste essentiellement à vérifier l-existence ainsi que l.état de conservation de ces plantes endémiques sur lesquelles s'est basée la définition de ces ZIP.

Malheureusement, les études récemment publiés sur la végétation dela région d'Oran (AinadTabet, 1988; Aimé, 1991; Hadjadj, 1995; Mansouri, 2012) ne rapportent pas beaucoup dinformations relatives à ce sujet et les données concernant la flore endémique locale demeurent ainsi rares voire inexistantes.

Par ailleurs, limpact de lsaction anthropique dévastatrice visible au niveau des habitats sauvages et naturelles de cette région (Mansouri, 2012; Bahi, 2012) réduit les chances de retrouver certains taxons endémiques et/ou rares comme Adenocarpus umbellatus, Bellevalia pomelii, Brassica spinescens, Silene auriculifoliaet Teucrium mauritanicum, sur lesquels la classification de cette région comme une ZIP a été basée.

Ainsi, cette étude vise à apporter plus d’éléments de connaissance sur cette flore endémiques rares et menacées.

En effet, le manque des données sur cette flore nous a conduits à se poser des questions par rapport au statut de conservation de ces plantes

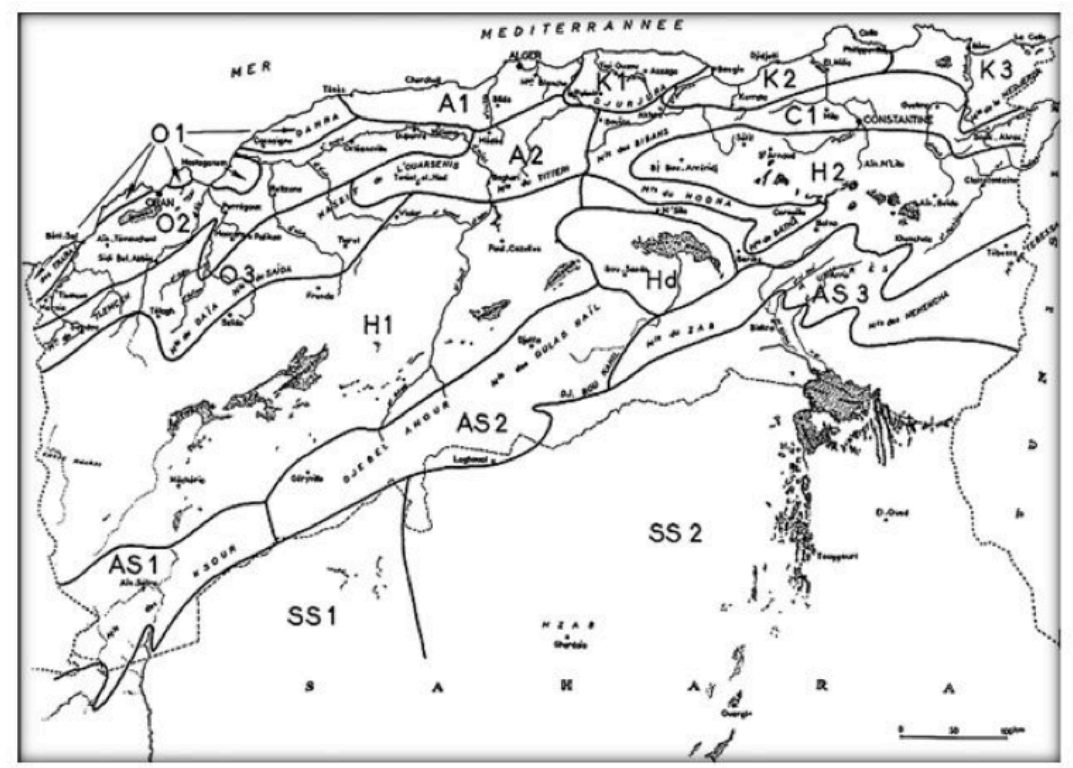

Figure 1. Carte de situation de la région d'étude au 1/500.000 : Extrait de la carte de découpage des sous-secteurs phytogéographiques du nord de l'Algérie (Quézel \& Santa, 1962-1963). 
endémiques.

A notre connaissance, aucune étude n>a portée sur la recherche et l, inventaire des plantes endémiques dans cette région.

Les résultats obtenus à 1,issue de cette exploration permettrons de connaitre la situation actuelle de ces plantes endémiques et aiderons a mieux définir leur statut de leur conservation.

\section{La zone d'etude}

La région étudiée est comprise entre le djebel Murdjadjo qui domine la ville d'Oran sur $580 \mathrm{~m}$ d'altitude à l'ouest et les monts d'Arzew à l'est 600 $\mathrm{m}$ d'altitude. Au sud la région est limitée par le mont Tessala $1061 \mathrm{~m}$ d'altitude et les Béni chougrane 910 m d'altitude.

Sur le plan phytogeographique, la région d'Oran comprend les secteurs biogeographiques O1 (sous-secteur des sahels littoraux oranais) et O2 (sous-secteur des plaines littorales oranaises) au sens de Quézel \& Santa (1962-63) (Fig. 1).

Sur le plan géologique la partie littorale de l'Algérie est la plus récente, la plus instable et là où il y a le plus de diversité de substrats géologiques. La région oranaise est donc très diversifiée, ses strates géologiques appartiennent aux quatres ères et sont de nature variés (Sadran,1982).

Sur le plan bioclimatique, la région d'Oran se situe au niveau de l'étage bioclimatique aride modéré à hiver froid (Emberger, 1942). Cette région à la particularité de posséder toutes les caractéristiques du climat méditerranéen et simultanément elle est soumise aux influences continentales (Meterfi et al., 2001).

D'après Mansouri (2012), la végétation locale étant très diversifiée, se compose de groupements de pelouses thérophytes et halophiles appartenant à plusieurs séries de végétation (Genévrier de Phénicie, Chêne liège, Thuya de Berbèrie, Chêne vert, Oléo-lentisque et Pin d'Alep).

\section{Materiel et methode}

La recherche des plantes endémiques a été entamée drabord par l,exploitation des documents de littérature notamment les flores d`Algérie de Battandier \&Trabut (1888-1890), Battandier \& Trabut (1895), Maire (1952-1987) et Quézel \& Santa (1962-63) afin de s'approprier un maximum dinformations sur les taxons endémiques notamment la localisation géographiques (lieu nommé, localité,...etc.) et la phénologie (période de floraison).

Quelques sorties de terrain ont été envisagées à cet effet ciblant la recherche de ces espèces au niveau du secteur (O1): Anthemis chrysantha,
Bellevalia pomeli, Brassica maurorum, Brassica spinescens, Carduus balansae, Helianthemum maritimum et Pulicaria filaginoides; Ainsi que dans le secteur (O2) : Najas arsenariensis, Spergularia doumerguaei, Atriplex chenopodioides, Erysimum semperflorens, Silene ghiarensis, Silene glaberrima et Teucrium santae.

Parallèlement, nous avons procédé à l'exploitation des données floristiques des 124 relevés phytoécologiques réalisés à travers la région d'Oran au niveau des 2 secteurs ( $\mathrm{O} 1$ et $\mathrm{O} 2$ ).

Ces relevés ont été effectués selon la méthode Sigmatiste de Braun-Blanquet et al. (1952) suivant un échantillonnage mixte (stratifié-aléatoire) au sens d'Aimé (1991). Ces relevés ont concernés les groupements de pelouse. La surface du relevé habituellement retenue au niveau de ces formations est de $1 \mathrm{~m}^{2}$ (Sekkal, 2007).

L'identification des espèces a été réalisée par le biais des flores suivantes : Coste (1937), Maire (1952) Quézel \& Santa (1962-1963).

L)actualisation des données de nomenclature et de chorologie a été effectuée suite à lsusage de l,index synonymique de la flore de l, Afrique du Nord (Dobignard \& Chatelain, 2010-13).

Les spécimens d/herbier constitués à l,issu de ce travail ont été déposés au niveau de l'herbier du Laboratoire d'Ecologie Végétale (Université Ahmed Ben Bella Oran1).

\section{Resultats}

\section{Inventaire}

La liste définitive des espèces endémiques retenues dans le présent travail a été arrêtée suite à plusieurs vérifications et recoupement des informations issues des flores et d'autres références comme El Oualidi et al. (2012).

Les résultats obtenus ont permis d'identifier 17 taxons endémiques au niveau de cette région (Tab.1).

Néanmoins, plusieurs taxons endémiques et rares n'ont pas été retrouvés au niveau des régions et/ou stations dans lesquelles ils ont été signalés dans la bibliographie. C'est le cas de Bellevalia pomelii, une endémiques très rare et localisée dans les monts de Murdjadjo. Cette région a connu beaucoup de modifications anthropiques notamment des constructions militaires ainsi que différentes formes de pollution de ces milieux forestiers et préforestiers. C'est également le cas pour: Anthemis chrysantha, Brassica maurorum, Carduus balansae, Pulicaria filaginoides, Najas arsenariensis, Spergularia doumerguaei, Atriplex chenopodioides, Erysimum semperflorens, Silene ghiarensis, Silene glaberrima et Teucrium santae. 


\begin{tabular}{|c|c|c|c|c|}
\hline Famille & Taxonsendémiques & $\begin{array}{c}\text { Chorologie Q } \\
\text { \& S 62-63 }\end{array}$ & $\begin{array}{l}\text { Chorologie El } \\
\text { Oualidi\& al } 2012\end{array}$ & $\begin{array}{l}\text { Chorologie D } \\
\& \text { Ch } 2013\end{array}$ \\
\hline Asteracea & Centaurea ferox Desf. & - & Alg & Alg \\
\hline Asteracea & Centaurea fragilis Durieu & Alg-Mar & Alg-Mar & Alg-Mar \\
\hline Asteracea & Centaurea involucrata Desf. & Alg-Mar & Alg-Mar & Alg-Mar \\
\hline Asteracea & Crepis salzmanni iBabc. & Alg-Mar & Mar & Alg-Mar \\
\hline Asteracea & $\begin{array}{l}\text { Coleostephus multicaulis } \\
\text { (Desf.) Durieu }\end{array}$ & Alg & Alg & Alg \\
\hline Asteracea & Sonchus mauritanicus Boiss.\&Reut. & N.A. & Mar-Alg-Tun & Mar-Alg-Tun \\
\hline Brassicaceae & Cordylo carpus muricatus Desf. & Alg-Mar & Alg-Mar & Alg-Mar \\
\hline Caryophyllaceae & Silene obtusifolia Willd. & Alg-Mar & - & Alg-Mar-Can \\
\hline Caryophyllaceae & Silene pomelii Batt. & Alg & Alg & Alg-Mar \\
\hline Cistaceae & Helianthemum maritimum Pomel & Alg & Alg & Alg \\
\hline Fabaceae & $\begin{array}{l}\text { Ononis antennata subsp. } \\
\text { natricoides Coss. ex Sirj. }\end{array}$ & Alg-Mar & Alg-Mar & Alg-Mar \\
\hline Fabaceae & Trifolium juliani Batt. & N.A. & Alg & Alg-Tun \\
\hline Frankeniaceae & $\begin{array}{l}\text { Frankenia laevis subsp. composita } \\
\text { (Pau \& Font Quer) Nègre }\end{array}$ & Paléo-Temp. & Mar & Alg-Mar \\
\hline Lamiaceae & Marrubium alyssoides Pomel & O2-O3 & - & Alg-Mar \\
\hline Lamiaceae & Thymus algeriensis Boiss \& Reut & N.A. & Mar-Alg-Tun-Lib & Mar-Alg-Tun-Lib \\
\hline Plantaginaceae & Linaria tingitana Boiss. \& Reut & Ibéro-Mar & Alg-Mar & Alg-Mar \\
\hline Plumbaginaceae & $\begin{array}{c}\text { Limonium battandieri Greuter \& } \\
\text { Burdet }\end{array}$ & Alg-Mar & Alg-Mar & Alg-Mar \\
\hline Plumbaginaceae & $\begin{array}{l}\text { Limonium cymuliferum (Boiss) } \\
\text { Sauvage \& Vindt }\end{array}$ & Alg-Mar & Alg-Mar & Alg-Mar \\
\hline Plumbaginaceae & Limonium duriaei (Girard) Kuntze & Alg-Mar & Alg-Mar & Alg-Mar \\
\hline
\end{tabular}

Tableau 1. Données chorologiques des taxons endémiques selon différentes références bibliographiques. Légende: Alg = endémique algérienne, Alg-Mar = endémique Algérie-Maroc, Alg-Tun = endémique Algérie-Tunisie, Magh = endémiqe Algérie- Maroc-Tunisie

Table 1. Chorological data of endemic taxa according to different bibliographical references. Legend: Alg = endemic Algerian, Alg-Ma = endemic Algeria-Morocco, Alg-Tun = endemic Algeria-Tunisia, Magh = endemic Algeria-MoroccoTunisia

\section{Chorologie}

Parmi les plantes identifiées, une majorité (10 taxons) ont conservés leurs types d'endémismes (Tab. 1). C'est-à-dire que leurs données chorologiques sont restées sans modification suite aux travaux de révisions récents. Le reste des espèces (6 taxons) ont subits des changements au niveau de leurs type d'endémismes. En effet, plusieurs espèces ayant connues une modification de leur chorologie suite à leurs découvertes dans des pays voisins et/ou dont la présence dans les pays dans lesquels ils ont été signalés au départ a été réfutée.

\section{Ecologie}

La majorité des espèces rencontrées suite à ce travail ont été retrouvées au niveau des pelouses et sables maritimes comme : Centaurea ferox, Centaurea fragilis, Coleostephu smulticaulis, Limonium Battandieri, Linaria tingitana et Silene obtusifolia (Tab.2).

D'autres taxons occupent plutôt des espaces forestiers et préforestiers au niveau des ouvertures bien exposés à la lumière dans les chaines de montagnes locales (Murdjadjo, Tessala) ou bien les forêts proches du littoral (forêt de Madagh) sur des substrats variables ( silices, calcaires, argiles,

Tableau 2. Ecologie et Biogéographie des taxons endémiques de la région d'Oran.

Table 2. Ecology and Biogeography of endemic taxa in the Oran region. 


\begin{tabular}{|c|c|c|c|}
\hline Taxons endémiques & Écologie (Sol et végétation) & $\begin{array}{l}\text { Biogeographie } \\
\text { Q \& S (1962-63) }\end{array}$ & $\begin{array}{l}\text { Lieu d'observation } \\
\text { (a Oran) }\end{array}$ \\
\hline Centaurea ferox Desf. & $\begin{array}{c}\text { Sable , Asteriscus maritimus, } \\
\text { Anacyclus clavatus, Linaria } \\
\text { tingitana, }\end{array}$ & $\begin{array}{c}\text { Localisée en Algérie } \\
\text { occidentale O1,O2,O3, } \\
\text { AS1 vient à l'Est jusqu'à } \\
\text { Affreville }\end{array}$ & Madaghplage \\
\hline Centaurea fragilis Durieu & $\begin{array}{l}\text { Sable, Anacyclus valentinus, Linum } \\
\text { usitatissimum, Stipa parviflora }\end{array}$ & $\begin{array}{l}\text { Dunes, rochers et } \\
\text { broussailles, ccc Algérie } \\
\text { occidentale }\end{array}$ & Fôret Madagh \\
\hline $\begin{array}{c}\text { Centaurea involucrata } \\
\text { Desf. }\end{array}$ & $\begin{array}{c}\text { Rocaille, bords de route, Centaurea } \\
\text { sphaerocephala, Plantago afra, Eruca } \\
\text { vesicaria, Ammoides pussilla }\end{array}$ & $\begin{array}{c}\text { pâturagearide, } \\
\mathrm{A} 1, \mathrm{~A} 2, \mathrm{H} 1, \mathrm{H} 2\end{array}$ & $\begin{array}{c}\text { Route vers salines d'arzew, } \\
\text { Bouyakour }\end{array}$ \\
\hline $\begin{array}{l}\text { Coleostephus multicaulis } \\
\text { (Desf.) Durieu }\end{array}$ & $\begin{array}{c}\text { Sables, Limonium sinuatum, Asteriscus } \\
\text { aquaticus, Echinops spinosus }\end{array}$ & O1-O2-O3-H1:Tiaret & Cap Falcon côtéphare \\
\hline $\begin{array}{c}\text { Cordylo carpusmuricatus } \\
\text { Desf. }\end{array}$ & $\begin{array}{c}\text { Affleurements calacires gréseux } \\
\text { devenant argileux en profendeur, } \\
\text { Hedypnois rhagadioloides, Ononis } \\
\text { reclinata, Bellardiatrixago, Geranium } \\
\text { lucidum }\end{array}$ & $\begin{array}{c}\text { O1,O2,O3- H1,H2 RR: } \\
\text { SS }\end{array}$ & SommetTessala \\
\hline Crepis salzmannii Babc. & $\begin{array}{l}\text { Substrat sableux , Silene fuscata, } \\
\text { Andryala floccosa, Hypochoeris } \\
\text { glabra, Plantago psyllium }\end{array}$ & R: littoral oranais & ForêtMadagh, cap Blanc \\
\hline $\begin{array}{c}\text { Frankenia laevis subsp. } \\
\text { composita (Pau \& Font } \\
\text { Quer) Nègre }\end{array}$ & $\begin{array}{c}\text { Roches maritimes, Coleostephus } \\
\text { multicaulis, Spergularia salina, } \\
\text { Asteriscus aquaticus, Echinops } \\
\text { spinosus }\end{array}$ & RR: O1 & Falaises cap Falcon \\
\hline $\begin{array}{l}\text { Helianthemum maritimum } \\
\text { Pomel }\end{array}$ & $\begin{array}{c}\text { Sol calcaire, Tuberaria guttata, } \\
\text { Aegilops triuncialis, Filago } \\
\text { pyramidata, } \\
\text { Paronychia argentea }\end{array}$ & Sable maritime, R: $\mathrm{O} 1$ & $\begin{array}{l}\text { Murdjadjo (antenne téléphonique) } \\
\text { descente vers Boutlelis }\end{array}$ \\
\hline $\begin{array}{l}\text { Limonium battandieri } \\
\text { Greuter\&burdet }\end{array}$ & $\begin{array}{l}\text { Sable, Silene nicaensis, Taraxacum } \\
\text { laevigatum, Urospermum picroides }\end{array}$ & $\begin{array}{l}\text { Bord de mer, Sebkha } \\
\text { juxtalittorale }\end{array}$ & Kristel en bord de mer \\
\hline $\begin{array}{l}\text { Limonium cymuliferum } \\
\text { (Boiss) Sauvage\&Vindt }\end{array}$ & $\begin{array}{l}\text { Bords de sebkhas et terrains salés, } \\
\text { Suaeda fruticosa, Frankenia } \\
\text { pulverulantae, Arthrocnemum } \\
\text { macrostachyum, Inula crtithmoides }\end{array}$ & CC O2-O3, AR /H1 & Lac de télamine \\
\hline $\begin{array}{l}\text { Limonium duriaei (Girard) } \\
\text { Kuntze }\end{array}$ & $\begin{array}{c}\text { Sebkha, Atriplex halimus, Suaeda } \\
\text { fruticosa, Lotus conrniculatus. } \\
\text { Plantago coronopus, Salicornia } \\
\text { Arabica }\end{array}$ & $\mathrm{O} 2-\mathrm{H} 1$ & La grande sebkha d'Oran \\
\hline $\begin{array}{l}\text { Linaria tingitana } \\
\text { Boiss.\&Reut }\end{array}$ & $\begin{array}{c}\text { Substrat siliceux, } \\
\text { Hippocrepis unisiliquosa, spergularia } \\
\text { salina, Crucianellamaritima, } \\
\text { Lotus creticus, }\end{array}$ & Sable maritime $A C: 01$ & Madaghplage \\
\hline $\begin{array}{l}\text { Marrubium alyssoides } \\
\text { Pomel }\end{array}$ & $\begin{array}{l}\text { Calendula arvensis, } \\
\text { Blackstoniaperfoliata }\end{array}$ & $\begin{array}{l}\text { Pasturages, } \\
\text { rocailles, R: dans } \\
\text { les confins algéro- } \\
\text { marocains }\end{array}$ & $\begin{array}{c}\text { Forêt ras el m'a Gdyel, corniche } \\
\text { supérieure (route des crêtes) } \\
\text { Kristel, Murdjadjo vers le port, cap } \\
\text { Blac, Madagh plage, }\end{array}$ \\
\hline $\begin{array}{c}\text { Ononis antennata } \\
\text { subsp. natricoidesCoss. } \\
\text { ex Sirj. }\end{array}$ & $\begin{array}{c}\text { Substrat marneux, Centaurea pullata, } \\
\text { Astragalus cruciatus, Geranium } \\
\text { robertianum }\end{array}$ & $\begin{array}{l}\text { Sables surtout maritimes } \\
\text { AR:O1-O2 }\end{array}$ & SommetTessala \\
\hline Silene obtusifolia Willd. & $\begin{array}{l}\text { Sol caillouteux, Calendula arvensis, } \\
\text { Teucrium capitatum, Evax pygmaea }\end{array}$ & $\begin{array}{l}\text { Sable et rochers } \\
\text { maritimes } A R: 01\end{array}$ & Cap Blanc \\
\hline Silene pomelii Batt. & - & $\begin{array}{l}\text { Forêt claire, pâturage } \\
\text { RC A1-O1-O2 }\end{array}$ & Aindefla (Kristel) \\
\hline $\begin{array}{l}\text { Sonchus mauritanicus } \\
\text { Boiss.\&Reut. }\end{array}$ & $\begin{array}{c}\text { Substrat calcaire, Inula viscosa, } \\
\text { Echium plantagineum, Ranaculus } \\
\text { batracoides }\end{array}$ & $\begin{array}{l}\text { RR: O2, Bord de la } \\
\text { sebkha }\end{array}$ & Cornichesupérieureversbredeah, \\
\hline $\begin{array}{l}\text { Thymus algeriensis } \\
\text { Boiss\&Reut }\end{array}$ & $\begin{array}{c}\text { Sol marneux, Teucrium capitatum, } \\
\text { Bombycelaena discolor }\end{array}$ & $\begin{array}{l}\text { CC dans toutes les régions } \\
\text { montagneuses, RR ailleurs }\end{array}$ & $\begin{array}{l}\text { Murdjadjo coté antennes, Tessala } \\
\text { centre insuffisance respiratoire }\end{array}$ \\
\hline Trifolium juliani Batt. & $\begin{array}{c}\text { Substrat caillouteux, Picris cupuligera, } \\
\text { Polygala mospeliaca, Crucianella } \\
\text { angustifolia }\end{array}$ & $\begin{array}{l}\text { CC K2-K3-C1, RR } \\
\text { ailleurs sahel Alger, } \\
\text { Santa Cruz Oran }\end{array}$ & $\begin{array}{l}\text { Corniche supérieure descente vers } \\
\text { boutlélis, reboisement Pin d'Alep }\end{array}$ \\
\hline
\end{tabular}


marnes,..etc.). II s;agit de: Centaurea fragilis, Cordylocarpus muricatus, Crepis salzmannii, Helianthemum maritimum, Marrubium alyssoides, Ononis antennata subsp. natricoides, Sonchus mauritanicus, Thymus algeriensis et Trifolium juliani.

Certaines espèces ont été rencontrées au niveau des rocailles et falaises rocheuses près de la mer comme Frankenia laevis subsp. Composita et Brassica spinescens, alors que d'autres sont essentiellement halophiles occupant les terrains salés près des Sebkhas (Limonium cymuliferum, Limonium duriael).

La végétation accompagnatrice est souvent constituée de plantes annuelles herbacées caractéristiques des pelouses, rocailles et falaises maritimes.

\section{Biogéographie}

La majorité de nos plantes endémiques ont été retrouvés au niveau du secteur O1. C'est-à-dire sur les côtes littorales d'Oran allant d'Arzew jusqu'aux frontières avec Ain Temouchent. Les plantes rencontrées dans le secteur $\mathrm{O} 2$ se présentaient essentiellement sur les monts de Tessala, Murdjadjou ainsi qu'au niveau de la Sebkha d'Oran (Tab.2).

\section{Discussion}

Les études portant sur l,inventaire de la flore endémiques en Algérie sont très rares. Quelques recherches récentes ont été publiées pour les régions de Hodna, I>Edough et Tiaret.

Zedam (2015) a pu compter 33 taxons endémiques dans chott el Hodna alors que Hamel et al. (2013) rapportent 31 taxons pour la péninsule de l'Edough. Dans la région de Tiaret, Miara el al. (2017) signalent 32 taxons endémiques. Le nombre de 17 taxons endémiques pour la région d'Oran parait plus ou moins faible comparé à ceux figurant dans les inventaires similaires dans d'autres régions du pays. Cela pourra être justifié d'une part par le type d'échantillonnage adopté dans cette étude qui a concerné essentiellement les terrains ouverts et les pelouses. D'autres part, beaucoup d'espèces n'ont pas été retrouvées à cause de l'action anthropique ayant fortement modifié les habitats naturelles de prédilection de ces plantes. Ainsi, ce premier inventaire demeure non-exhaustive et besoin d'être poursuivis par la recherches des espèces manquantes, mais aussi par l'échantillonnage d'autres strates de végétation notamment les forêts et les matorrals.

Sur le plan chorologique, plusieurs taxons ont conservé leur endémisme sans changements:
Sonchus mauritanicus figurant comme une endémique de l'Afrique du Nord (Quézel \& Santa, 1962-63) est retenue dans les références les plus récentes comme une endémique Algérie, Maroc et Tunisie (Dobignard \& Chatelain, 2010-13; El Oualidi et al., 2012).

Cordylocarpus muricatusest a été signalé comme une endémique Algéro-marocaine au niveau de références consultés (Quézel \& Santa, 1962-63; El Oualidi et al., 2012; Dobignard \& Chatelaîn, 2010-13).

Crepis salzmanni figure comme endémique Algérie-Maroc au niveau des principales références (Quézel \& Santa, 1962-63; Dobignard \& Chatelain, 2010-13). Néanmoins, El Oualidi et al. (2012) rapportent l'existence de cette plante uniquement au Maroc.

Centaurea fragilis n'a pas changé de statut chorologique d'endémique Algérie-Maroc.

Coleostephus multicaulis est une endémique stricte de l'Algérie commune dans la région de l'Oranie.

Centaurea ferox figure comme une endémique algérienne stricte dans la flore d'Algérie de Quézel \& Santa (1962-63). Son statut chorologique n'a pas connu de changements suite aux travaux de révision récents (El Oualidi et al., 2012; Dobignard \& Chatelain, 2010-13).

Limonium battandieri n'a pas changé de statut d'endémique Algérie-Maroc.

De même, Limonium duriaei a gardé son statut d'endémique Algérie-Maroc.

Ononisantennatasubsp. natricoides est citée comme endémique Algérie-Maroc au niveau des différentes références consultées (Quézel \& Santa, 1962-63; El Oualidi et al., 2012; Dobignard \& Chatelain, 2013).

Aussi, Helianthemum maritimum n'a pas changé de statut d'endémique algérienne stricte. D'après Meddour (2012), cette plante est l'une des plus particulières du secteur (O1) lui conférant une forte individualité floristique.

D'autres espèces ont changées de type d'endémisme comme Silene pomelii qui a été mentionnée comme une endémique algérienne stricte par Quézel \& Santa (1962-63) et El Oualidi et al. (2012). Or, cette plante a été retenue comme une endémique Algérie-Maroc par Dobignard \& Chatelain (2010-13).

Frankenia laevis subsp. composita ne figure pas comme endémique dans la flore de Quézel \& Santa (1962-63). Elle est retenue comme une endémique Marocaine par El Oualidi et al. (2012). Dobignard \& Chatelain (2010-13) mentionnent ce taxon comme endémique Algérie-Maroc.

Linaria tingitana figure dans la flore de Quézel 
\& Santa (1962-63) comme Ibéro-Marocaine. Elle figure dans les références plus récentes comme une endémique Algérie-Maroc (El Oualidi et al., 2012; Dobignard \& Chatelain, 2010-13).

Sur le plan écologique, nos observations relatives aux conditions stationnelles dans lesquelles les espèces endémiques ont été retrouvées sont dans l'ensemble en accord avec Quézel \& Santa (1962-63).

Quelques espèces ont été néanmoins retrouvées dans des milieux non indiqués dans cette références à lsimage de Sonchu smauritanicus ou Helianthemum maritimum retrouvés sur substrats calcaire. Le reste des endémiques sont en majorité des plantes des sables maritimes pouvant parfois pénétrer jusqu'à l'Atlas tellien occidentale comme Coleostephus multicaulis qui caractéristique de plusieurs groupements végétaux décrits récemment dans la région de l'Oranie (Mansouri, 2012; Miara, 2017). Cette plante semble privilégier les sols sableux au niveau des ouvertures préforestieres des chênaies vertes dans l'Atlas tellien occidentale de Tiaret (Miara, 2017).

Sur le plan biogéographique les données concernant la répartition de nos espèces sont en accord avec les observations de Quézel \& Santa (1962-63).

Par ailleurs, plusieurs espèces ne figurent pas dans les inventaires récents publiés pour le secteur de l'Oranie (O1, $\mathrm{O} 2$ et O3) à l'image de Centaurea ferox, Helianthemum maritimum, Linaria tingitana, Limonium battandieri, Limonium cymuliferum, Limonium duriaei,

Marrubium alyssoides, Ononis antennata subsp. natricoides et Silene obtusifolia.

Or, des taxons comme: Centaurea fragilis, Centaurea involucrata, Crepis salzmannii et Frankenia laevis subsp. composita ont été signalées par Medjahdi et al. (2009) au niveau des monts de Traras (O1) dans la région de Tlemcen.C'est également le cas de Cordylocarpus muricatus qui a été également signalé dans la région de Tiaret par Miara et al. (2017).

Silene pomelii a été signalée dans la région de Tlemcen par Bouazza et al. (2001). Coleostephus multicaulis ont été retrouvé dans la région de Tlemcen (Leutreuch-Belarouci et al., 2009) ainsi que dans la région de Tiaret (Miara et al., 2017). Quant à Sonchus mauritanicus, il a été récemment vu dans la région de la Macta par Megharbi et al. (2016). Enfin, Miara et al. (2017) signalent Thymus algeriensis dans le secteur atlasique de Tiaret.

\section{Conclusion}

Cette étude constitue une première prospection de la flore endémique de la région d`Oran depuis I, indépendance du pays en 1962. Les données obtenues suite à ces recherches renforcent les inquiétudes par rapport au statut de conservation des plantes endémiques de ce secteur. En effet, à lexception de Helianthemum maritimumet Brassica spinescens qui ont été observés dans la présente recherche, un nombre important des espèces endémiques notamment celles endémiques et rares comme Pulicaria filaginoides et Bellevalia pomeli possédant un grand intérêt biologique et patrimoniale (Yahi et al., 2012) n'ont pas été retrouvés, et cela malgré nos efforts.

D'autres recherches seront à envisager dans l'avenir afin de déterminer l'état de conservation ou de l'existence de ces espèces, alors que nous soutenons fortement l'hypothèse de la disparition de certains de ces taxons notamment les plus rares.

\section{Bibliographie}

Aimé, S. (1991). Étude écologique de la transition entre les bioclimats subhumide, semi-aride et aride dans l'étage thermo-méditerranéen du tell oranais (Algérie occidentale). Thèse doct. Etat, Univ. Aix Marseille III, $156 p+a n n$.

Ainad-Tabet, L. (1988). Étude d'un échantillon représentatif des pelouses d'Oranie en relation avec les conditions de sol .Mém. Magister, Univ. Oran, Sénia, 180p.

Bahi, K. (2012). Contribution à l'étude phytoécologique des zones humides de la région d'Oran, Mém. Mag. Univ. Oran, 118p.

Battandier, J.A. \& L.C. Trabut. $(1888,1890)$. Flore d'Algérie. Adolphe Jourdan (éd), Alger, 825p.

Battandier, J.A. \& L.C. Trabut. (1895). Flore d'Algérie (Monocotyledones). Typographie Adolphe Jourdan (éd), Alger, 256p

Bouazza, M., M. Mahboubi., R. Loisel \& N. Benabadji. (2001). Bilan de la flore de la région de Tlemcen (Oranie - Algérie). Forêt Méditerranéenne, T. XXII, $\mathrm{n}^{\circ}$ 2: $130-136$.

Braun-Blanquet, J., N. Roussine \& R. Nègre. (1952). Les groupements végétaux de la France méditerranéenne. Dir. Carte Group. Vég. Afr. Nord, CNRS, 292 p.

Coste, H. (1937). Flore descriptive et illustré de la France, de la Corse et des contrées limitrophes. Librairie des Sciences et des Arts (éd), Paris, 3 tomes.

Dobignard, A. \& C. Chatelain. (2010-2013). Index synonymique de la flore d'Afrique du Nord: Pteridophyta, Gymnospermae, Monocotyledoneae, Conservatoire et Jardin botaniques de la Ville de Genève (éd), 5 vol, 2236p.

El Oualidi, J., H. Khamar., M. Fennane., M. Ibn Tattou., S. Chauvet. \& M.S. Taleb. (2012). Checklist des endémiques et spécimens types de la flore vasculaire de l'Afrique du Nord. Doc Inst Sci. Universite mohammed v-agdal, rabat, $n^{\circ} 25,192 \mathrm{p}$.

Greuter, W. (1995). Origin and peculiarities of 
Mediterranean island floras. EcologiaMediterranea, 21 (1-2): 1-10.

Hadjadj-Aoul, S. (1995). Les peuplements du Thuya de Berbèrie (Tetraclinisarticulata, Vahl, Master) en Algérie: phytoécologie, syntaxonomie et potentialités sylvicoles. Thèse Doc. Univ. Aix-Marseille III, 160p + ann.

Hamel, T., R. Seridi., G. De Belair., A. Slimani. \& B. Babali. (2013). Flore vasculaire rare et endémique de la péninsule de l'Edough (Nord-Est algérien). Rev. Sci. Technol., Synthèse. 26: 65 - 74.

Heywood, V.H. (1998). The Mediterranean region a major centre of plant diversity. Ed. Wild food and nonfood plants information networking. Cahiers Options Méditerranéens, V (3): 5-15.

Letreuch-Belarouci, A., B. Medjahdi., N. LetreuchBelarouci.\& K. Benabdeli. (2009). Diversité floristique des subéraies du parc national de Tlemcen (Algérie). Act. Bot. Malac., 34 : 77-89.

Maire, R. (1952-1987). Flore de l'Afrique du Nord. Le chevalier (éd), Paris, 16 Vol.

Mansouri, S. (2012). Les pelouses de la région d'Oran : Flore et phytoécologie. Mém. Mag, Univ. Oran, 92p.

Meddour, R. (2010). Bioclimatologie, phytogéographie \& phytosociologie en Algérie ( exemple des groupements forestiers et preforestiers en Kabylie Djurdjuréenne. Thèse Doc, Univ. Mouloud Maameri Tizi-Ouzou, 397p.

Megharbi, A., F. Abdoun, B. Belgherbi. (2016). Diversité floristique en relation avec les gradients abiotiques dans la zone humide de la Macta (ouest d'Algérie). Revue d'Ecologie (Terre et Vie), Vol. 71 (2): 142-155.

Meterfi, B., N. Leutreuch-Belarouci, K. Benabdelli. (2002). Incidence de la fluctuation des précipitations sur l'occupation des sols dans les hautes plaines de Sidi Belabess, Algérie. Rev. Physio-Geo. 65p.

Miara, M.D., M. Ait Hammou, K. Rebbas, H. Bendif. (2017). Flore endémique, rare et menacées de l'Atlas tellien occidental de Tiaret (Algérie). Acta Botanica Malacitana, 42 (2): 271-285. DOI: http://dx.doi. org/10.24310/abm.v42i2.3590

Mittermeier, R.A., N. Myers, J.B. Thomsen, G.A. Da Fonseca \& S. Olivieri. (1998). Biodiversity hotspots and major tropical wilderness areas: approaches to setting conservation priorities. Conservation Biology, vol. 12 (3).

Myers, N. (1988). Threatened biotas: Hotspots in tropical forests. Environmentalist. 8, 178-208.

Quézel, P. \& F. Médail. (2003). Écologie et Biogéographie des forêts du bassin méditerranéen. Elsevier (éd), Paris, $571 \mathrm{p}$.

Quézel, P. \& S. Santa. (1962). Nouvelle flore de l'Algérie et des régions désertiques méridionales. CNRS (éd), Paris, 2 Volumes.

Quézel, P. (2000). Réflexions sur l'évolution de la flore et de la végétation au Maghreb méditerranéen. Ibis presse (éd), 133p.

Quézel, P. (1956). Contribution à l'étude des forêts de chênes à feuilles caduques d'Algérie. Mém. Soc. Hist.Nat. Afr. Nord, N.S, Alger, 1, 1-57.

Quézel, P. (1985). Definition of the Mediterranean region and the origin of its flora.In: Gomez-Campo C. (éd.), Plant Conservation in the Mediterranean area. Dr. W. Junk Publ., Dordrecht, 9-24pp.

Rivas-Martínez, S. \& J. Loidi Arregui. (1999). Bioclimatology of the Iberian Peninsula. Itin. Geobot, 13, 41-47.

Sadran, G. (1982). Les roches cristallines du littoral oranais. XIXe congrès géologique international. Monographies régionales, première série, Alger,18p.

Sekkal, F.Z. (2007). Essai de caractérisation phytoécologique des pelouses dans les monts de Traras. Mém. Magister, Univ. Oran, 100p.

Véla, E. \& S. Benhouhou. (2007). Évaluation d'un nouveau point chaud de biodiversité végétale dans le Bassin méditerranéen (Afrique du Nord). Comptes rendus Biologies, 330 : 589-605.

Walter, K.S. \& H.J. Gillett. (1997- 1998). IUCN Red List of Threatened Plants. Compiled by the World Conservation Monitoring Centre. IUCN - The World Conservation Union, Gland, Switzerland and Cambridge, UK, 336p.

Yahi, N., E. Véla, S. Benhouhou, G. De Belair \& R. Gharzouli. (2012). Identifying Important Plants Areas (Key Biodiversity Areas for Plants) in northern Algeria. Journal of Threatened Taxa, 4 (8): 2753-2765.

Zedam, A. (2015). Etude de la flore endémique de la zone humide du Chott El Hodna, inventaire et préservation. Thèse Doct, Université de Sétif, Algérie, 368p. 